\title{
Kerker Conditions upon Lossless, Absorption, and Optical Gain Regimes
}

\author{
Jorge Olmos-Trigo $\odot,{ }^{1, *}$ Cristina Sanz-Fernández $\odot,{ }^{2}$ Diego R. Abujetas $\odot,{ }^{1,3}$ Jon Lasa-Alonso $\odot,{ }^{1,2}$ Nuno de Sousa $\odot,{ }^{1}$ \\ Aitzol García-Etxarri๑, ${ }^{1,2}$ José A. Sánchez-Gil ${ }^{3}{ }^{3}$ Gabriel Molina-Terriza $\odot^{1,2,4}$ and Juan José Sáenz ${ }^{1,4}$ \\ ${ }^{1}$ Donostia International Physics Center (DIPC), 20018 Donostia-San Sebastián, Basque Country, Spain \\ ${ }^{2}$ Centro de Física de Materiales (CFM-MPC), Centro Mixto CSIC-UPV/EHU, 20018 Donostia-San Sebastián, Spain \\ ${ }^{3}$ Instituto de Estructura de la Materia (IEM-CSIC), Consejo Superior de Investigaciones Científicas, Serrano 121, 28006 Madrid, Spain \\ ${ }^{4}$ IKERBASQUE, Basque Foundation for Science, 48013 Bilbao, Spain
}

(Received 5 May 2020; accepted 6 July 2020; published 13 August 2020)

\begin{abstract}
The directionality and polarization of light show peculiar properties when the scattering by a dielectric sphere can be described exclusively by electric and magnetic dipolar modes. Particularly, when these modes oscillate in phase with equal amplitude, at the so-called first Kerker condition, the zero optical backscattering condition emerges for nondissipating spheres. However, the role of absorption and optical gain in the first Kerker condition remains unexplored. In this work, we demonstrate that either absorption or optical gain precludes the first Kerker condition and, hence, the absence of backscattered radiation light, regardless of the particle's size, incident wavelength, and incoming polarization. Finally, we derive the necessary prerequisites of the second Kerker condition of the zero forward light scattering, finding that optical gain is a compulsory requirement.
\end{abstract}

DOI: 10.1103/PhysRevLett.125.073205

In 1983, Kerker, Wang, and Giles predicted that, under plane wave illumination, magnetic spheres with equal relative permittivity $\epsilon$ and permeability $\mu$ radiate no light in the backscattering direction [1]. They also concluded that if $\epsilon=(4-\mu) /(2 \mu+1)$ for nanospheres, this zero optical light scattering condition happened in the forward direction.

Three decades later, a renewed version of these ideas was proposed for subwavelength dielectric spheres $(\mu=1)$ of high refractive index (HRI) materials [2], reinvigorating the interest on these light scattering conditions. Notably, the scattering properties of HRI nanospheres can be fully described by dipolar modes via the first electric and magnetic Mie coefficients, without a spectral overlap from higher-order modes for certain ranges of the electromagnetic (EM) spectrum [3,4]. In terms of the electric and magnetic scattering phase shifts [5], these coefficients generally read as

$$
a_{l}=i \sin \alpha_{l} e^{-i \alpha_{l}}, \quad b_{l}=i \sin \beta_{l} e^{-i \beta_{l}},
$$

respectively, where $\alpha_{l}$ and $\beta_{l}$ are real in the absence of losses or optical gain.

At the first Kerker condition [2], given by $\alpha_{1}=\beta_{1} \Leftrightarrow$ $a_{1}=b_{1}$, the electric and magnetic dipolar modes oscillate in phase with equal amplitude. This optical response drives to destructive interference between the scattered fields at the backscattering direction, which is commonly referred to as zero optical backscattering condition [1]. This anomalous light scattering condition was first experimentally measured in the limit of small particle in the microwave regime for ceramic spheres [6] and, soon after, in the visible spectral range for HRI Si [7] and GaAs nanospheres [8]. However, recent results suggest that the concept of small particle is sufficient, but not necessary, to guarantee a dipolar response in the optical scattering of an object [9]. Consequently, the aforementioned backscattering anomalies could also be measured on larger dielectric particles. Interestingly, the absence of backscattered light emerges at the first Kerker condition for dipolar particles regardless of the incoming polarization [10-13]. On the other hand, for cylindrically symmetric particles, the absence of backscattered light follows from the preservation of EM helicity [14-18]. Conservation of helicity has proven crucial in many applications such as enhanced chiral light-matter interactions [19-25], or in the spin-orbit interactions of light [26-32]. In this vein, it has been reported that from a relatively simple far-field measurement of the EM helicity at a right angle, the radiation pattern of the dipolar particle is inferable [33]. This phenomenon arises since the asymmetry parameter $(g)$, which encodes the particle's optical response, is equivalent to the EM helicity at the direction perpendicular to the incoming wave when the object is excited by a beam with well-defined helicity $(\sigma= \pm 1)$, namely, $\Lambda_{\pi / 2}=2 \sigma g$ [33]. This relation straightforwardly links the EM helicity with the $g$ parameter, which appears in multiple branches of physics such as optical forces [34-36], light transport phenomena [37-39], or wavelength-scale errors in optical localization [40]. Remarkably, this wavelength's error limit can be drastically surpassed at the first Kerker condition for dipolar particles, where an optical vortex arises in the backscattering region [41]. 
In contrast to the first Kerker condition, the zero optical scattering condition in the forward direction, given for dipolar particles by $a_{1}=-b_{1}$, is precluded by the optical theorem for lossless spheres [42-44]. As an alternative, the generalized second Kerker condition (GSKC), mathematically expressed for dipolar particles as $\alpha_{1}=-\beta_{1}$, was proposed as an approximated condition for nondissipating spheres that might guarantee the maximum backwardforward scattering ratio while respecting energy conservation [2]. Indeed, the GSKC is the optimal backward light scattering condition; however, it does not generally imply a nearly zero optical forward scattering [45], contrary to what could be expected from previous works [2,46-48].

Most research on these topics is dedicated to the optical response of lossless HRI nanospheres in the dipolar regime. However, the role of absorption and optical gain, which has been considerably discussed in the so-called Mie theory framework during the last century, remains unexplored in the context of the abovementioned first and second Kerker conditions [49]. In this work, we analytically demonstrate that either losses or optical gain inhibit the first Kerker condition for homogeneous spheres regardless of the particle's size, incident wavelength, incoming polarization, and multipole order. Hence, dissipating spheres such as dielectric Mie spheres in the visible spectral range and plasmonic particles, such as metal spheres, cannot exhibit the first Kerker condition. These results unveil a hidden connection between two symmetries: energy conservation, mathematically expressed in terms of the optical theorem, and the EM duality, which is restored at the first Kerker condition. As a result, we show that the EM helicity cannot be preserved after scattering by an arbitrary dielectric sphere in the presence of losses or optical gain. Hence, neither can the zero optical backscattering condition be fulfilled in that scenario. In particular, for a Germanium (Ge) sphere in the dipolar regime, we quantify the gradual drift from the ideal zero optical backscattering condition as the absorption rate is increased. Finally, we prove that optical gain is mandatory to reach the zero forward light scattering condition.

Mie theory [5] gives an exact solution of Maxwell's equations for a spherical particle in a homogeneous medium under plane wave illumination. It allows writing the extinction, scattering, and absorbing efficiencies of the particle as

$Q_{\mathrm{ext}}=\frac{2}{x^{2}} \sum_{l=1}^{\infty}(2 l+1) \Re\left\{a_{l}+b_{l}\right\}=\sum_{l=1}^{\infty}\left(Q_{\mathrm{ext}}^{a_{l}}+Q_{\mathrm{ext}}^{b_{l}}\right)$,

$Q_{\text {sca }}=\frac{2}{x^{2}} \sum_{l=1}^{\infty}(2 l+1)\left(\left|a_{l}\right|^{2}+\left|b_{l}\right|^{2}\right)=\sum_{l=1}^{\infty}\left(Q_{\text {sca }}^{a_{l}}+Q_{\text {sca }}^{b_{l}}\right)$,

where $Q_{\mathrm{abs}}=Q_{\mathrm{ext}}-Q_{\mathrm{sca}}$.
The efficiencies are dimensionless magnitudes given by the ratio between the cross section and the geometrical area, $Q=\sigma / \pi R^{2}$, where $R$ is the radius of the particle. Here, $x=k R$ is the size parameter, $k=m_{h} k_{0}=m_{h}(2 \pi) / \lambda_{0}, \lambda_{0}$ being the wavelength in vacuum and $m_{h}$ the refractive index of the external medium. The Mie coefficients, $a_{l}$ and $b_{l}$, can be expressed in terms of the scattering phase shifts [see Eq. (1)] by [5],

$$
\begin{aligned}
& \tan \alpha_{l}=-\frac{S_{l}^{\prime}(m x) S_{l}(x)-m S_{l}(m x) S_{l}^{\prime}(x)}{S_{l}^{\prime}(m x) C_{l}(x)-m S_{l}(m x) C_{l}^{\prime}(x)}, \\
& \tan \beta_{l}=-\frac{m S_{l}^{\prime}(m x) S_{l}(x)-S_{l}(m x) S_{l}^{\prime}(x)}{m S_{l}^{\prime}(m x) C_{l}(x)-S_{l}(m x) C_{l}^{\prime}(x)} .
\end{aligned}
$$

Here, $m=m_{p} / m_{h}$ is the refractive index contrast, where $m_{p}$ is the refractive index of the particle while $S_{l}(z)=$ $\sqrt{\pi z / 2} J_{l+1 / 2}(z)$ and $C_{l}(z)=\sqrt{\pi z / 2} N_{l+1 / 2}(z)$ are the Riccati-Bessel functions, where $J_{l+1 / 2}(z)$ and $N_{l+1 / 2}(z)$ are the Bessel and Neumann functions, respectively.

According to Eqs. (4) and (5), the first Kerker condition, in which the electric and magnetic dipolar modes oscillate in phase with identical amplitude, can be obtained either when $S_{1}(m x)=0$ (nodes of first kind) or when $S_{1}^{\prime}(m x)=0$ (nodes of second kind) [5]. However, for complex values of the refractive index contrast [50], i.e., $\Im\{m\} \neq 0$, which corresponds either with absorption $(\Im\{m\}>0)$ or active media $(\Im\{m\}<0)$, these nodes are unreachable. We will prove this, and generalize it for arbitrary multipolar modes, using the following Lemmas:

(1) When $v>-1$ the zeros of $J_{v}(z)$ are all real [51].

(2) When $v>-1$ and $a, b \in \mathbb{R}$, then $a J_{v}(z)+b z J_{v}^{\prime}(z)$ has all its zeros real, except when $a / b+v<0$ [51].

Lemma 1 directly implies that the node of the first kind, $S_{l}(m x)=0$, is inhibited for spheres with either gain or loss since the zeros of the Bessel functions occur for exclusively real arguments, while in these cases $\Im(m) \neq 0$. On the other hand, the node of the second kind, given by

$$
S_{l}^{\prime}(m x)=J_{l+1 / 2}(m x)+2 m x J_{l+1 / 2}^{\prime}(m x)=0,
$$

cannot be satisfied for $\Im\{m\} \neq 0$, since following Lemma 2 with $a=1, b=2$, and $v=l+\frac{1}{2}$ the condition $a / b+$ $v<0$ is inaccessible because $l \geq 1$ [5].

The immediate physical consequence of these lemmas is straightforward: either absorption or optical gain inhibits the emergence of the first Kerker condition. It is important to note that the validity of these conclusions holds regardless of the particle size, incident wavelength, (complex) refractive index contrast, and polarization of the incoming light. Remarkably, this result is valid for any multipole order $l$. In short, we can conclude that $a_{l} \neq b_{l} \forall l$ when $\Im\{m\} \neq 0$, making this demonstration general. 
Interestingly, these conclusions can also be understood by analyzing the extinction and scattering efficiencies arising from electric and magnetic modes. In the presence of losses or gain, the extinction and scattering efficiencies of an arbitrary electric multipole $l$ cannot be identical to the magnetic counterpart of the same multipole $l$. According to the right-hand side of Eqs. (2) and (3), this phenomenon implies the following: Taking into account that in the presence of gain or losses $Q_{\mathrm{abs}} \neq 0$, if $Q_{\mathrm{sca}}^{a_{l}}=Q_{\text {sca }}^{b_{l}}$ then $Q_{\text {ext }}^{a_{l}} \neq Q_{\text {ext }}^{b_{l}}$. These relations imply that if $\Im\{m\} \neq 0$ the electric and magnetic modes cannot simultaneously oscillate in phase with equal amplitude, unveiling a connection between the first Kerker condition and energy conservation.

To get a deeper insight into these results, let us calculate the expected value of EM helicity after scattering. The scattered fields outside the sphere, decomposed in components of well-defined EM helicity [52], i.e., $\mathbf{E}_{\text {sca }}=\mathbf{E}_{\text {sca }}^{+}+$ $\mathbf{E}_{\text {sca }}^{-}$with $\Lambda \mathbf{E}_{\text {sca }}^{\sigma}=\sigma \mathbf{E}_{\text {sca }}^{\sigma}$, can be written in terms of "outgoing" vector spherical wave functions, $\Phi_{l m}^{\sigma^{\prime}}$ (defined in [15]) as

$$
\begin{gathered}
\mathbf{E}_{\mathrm{sca}}^{\sigma}=E_{0} \sum_{l=1}^{\infty} \sum_{m=-l}^{+l} D_{l m}^{\sigma} \Phi_{l m}^{\sigma}, \\
\left(\begin{array}{c}
D_{l m}^{+} \\
D_{l m}^{-}
\end{array}\right)=-\left(\begin{array}{cc}
{\left[a_{l}+b_{l}\right]} & {\left[a_{l}-b_{l}\right]} \\
{\left[a_{l}-b_{l}\right]} & {\left[a_{l}+b_{l}\right]}
\end{array}\right)\left(\begin{array}{c}
C_{l m}^{+} \\
C_{l m}^{-}
\end{array}\right), \\
Z \mathbf{H}_{\mathrm{sca}}^{\sigma}=-i \Lambda \mathbf{E}_{\mathrm{sca}}^{\sigma},
\end{gathered}
$$

where $C_{l m}^{\sigma}$ are the expansion coefficients of the incoming wave in a basis of vector spherical harmonics.

Under illumination by a circularly polarized plane wave with well-defined helicity $(\sigma= \pm 1)$ and angular momentum in the wave's propagation direction $J_{z}=m=\sigma$ [15], it can be shown that the expected value of the EM helicity is given by [9]

$$
\begin{aligned}
\langle\Lambda\rangle & =\frac{\int \mathbf{E}_{\mathrm{sca}}^{*} \cdot \boldsymbol{\Lambda} \mathbf{E}_{\mathrm{sca}} d \boldsymbol{\Omega}}{\int \mathbf{E}_{\mathrm{sca}}^{*} \cdot \mathbf{E}_{\mathrm{sca}} d \boldsymbol{\Omega}} \\
& =2 \sigma\left[\frac{\sum_{l=1}^{\infty}(2 l+1) \Re\left\{a_{l} b_{l}^{*}\right\}}{\sum_{l=1}^{\infty}(2 l+1)\left(\left|a_{l}\right|^{2}+\left|b_{l}\right|^{2}\right)}\right] .
\end{aligned}
$$

From Eq. (10) it is straightforward to notice that in the presence of gain or losses, since $a_{l} \neq b_{l} \forall l$, the EM helicity is not preserved, namely, $|\langle\Lambda\rangle| \neq 1$.

Figure 1 summarizes quantitatively this conclusion for a Ge sphere of different radii. For $\Im\{m\}>0$, corresponding to the visible spectral range [see Fig. 1(a)], the EM helicity is far from being preserved, regardless of the size of the Ge sphere in the entire visible spectral range which corresponds to the dashed rectangle in Fig. 1(b). This phenomenon can be inferred from $\langle\Lambda\rangle_{\max }$, which is obtained by finding for each of the incident wavelengths, the radius that

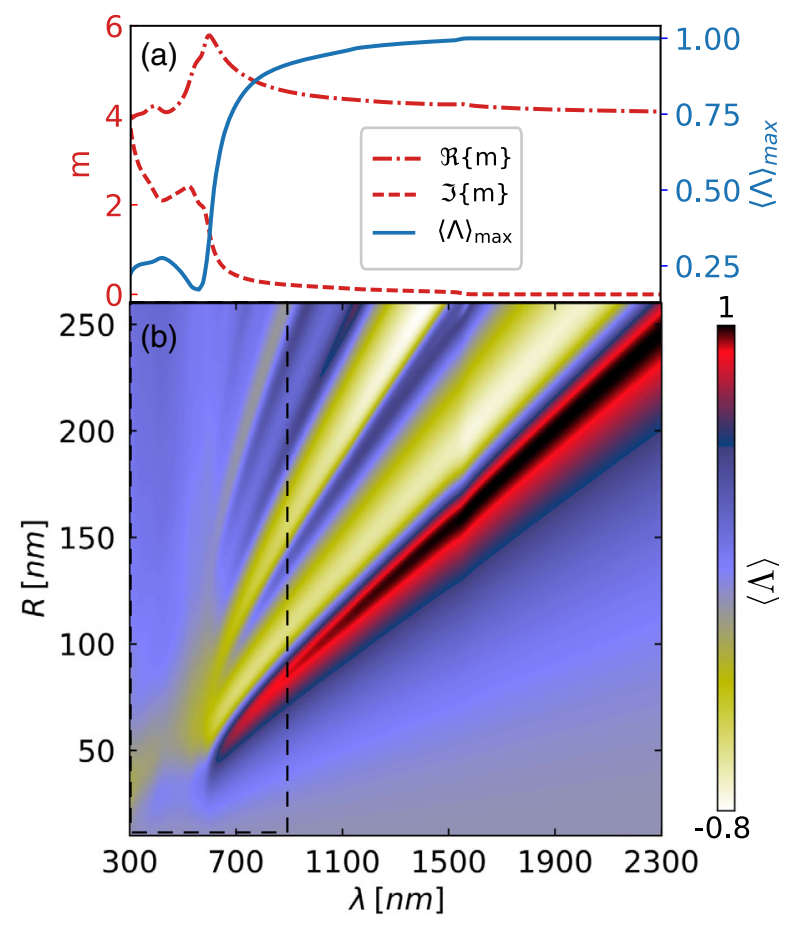

FIG. 1. (a) Real (dash-dotted red) and imaginary part (dashedred) of the refractive index contrast $(m)$ vs the incident wavelength $(\lambda)$ for a Ge sphere. Maximum of the expected value of the EM helicity in solid blue, $\langle\Lambda\rangle_{\max }$, for a Ge sphere vs $\lambda$ under plane wave illumination with $\sigma=+1$. (b) Color map of $\langle\Lambda\rangle$ vs $\lambda$ and particle's size $(R)$ for a Ge sphere under plane wave illumination with $\sigma=+1$. The visible range is encompassed by a dashed rectangle. As mentioned in the text, in this region helicity conservation is never fulfilled.

maximizes the EM helicity. To the contrary, in the telecom spectral range, where losses are negligible [see Fig. 1(a)], the maximum value of the EM helicity is preserved at the first Kerker condition, $\langle\Lambda\rangle_{\max } \approx 1$.

To get insight into the relevance of the breaking of the first Kerker condition due to absorption effects in the scattering radiation pattern, let us now consider the differential scattering cross section [5],

$$
\frac{d \sigma_{\mathrm{s}}}{d \Omega}=\lim _{k r \rightarrow \infty} r^{2} \frac{\mathbf{S} \cdot \hat{\mathbf{r}}}{S_{0}}
$$

Here, $\mathbf{S}=\Re\{\mathbf{E} \times \mathbf{H}\} / 2$ denotes the scattered Poynting vector, $S_{0}$ refers to the amplitude of the incoming Poynting vector amplitude, and $\hat{\mathbf{r}}$ is the radial unit vector. By taking into account Eqs. (7)-(9), when just retaining the dipolar contribution $(l=1)$, it can be shown that the (integralnormalized) differential scattering cross section reads as

$$
\frac{d \sigma_{\mathrm{s}}}{d \Omega}=\frac{3}{8 \pi}\left(\frac{1+\cos ^{2} \theta}{2}+2 g \cos \theta\right),
$$




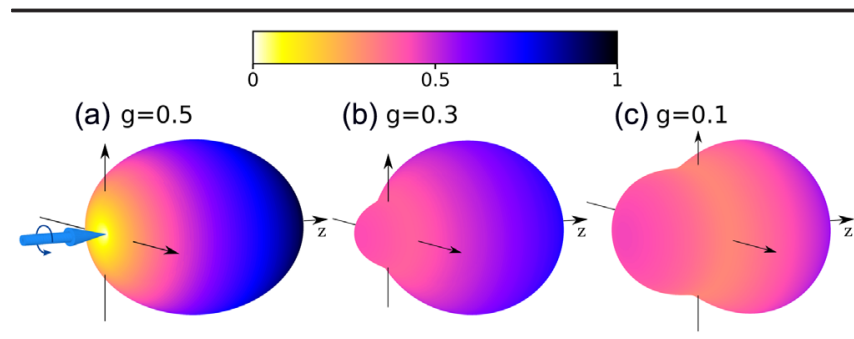

FIG. 2. Normalized scattering patterns by Ge spheres calculated from Eq. (12) in the telecom spectral regime (a) $[\lambda=2100 \mathrm{~nm}$ and $R=223 \mathrm{~nm}]$ and visible spectral range (b) $[\lambda=632 \mathrm{~nm}$ and $R=48 \mathrm{~nm}]$ and (c) $[\lambda=575 \mathrm{~nm}$ and $R=35 \mathrm{~nm}]$. The $g$ parameter is given by $g=0.5,0.3,0.1$, respectively, in the dipolar regime. The color map and the $3 \mathrm{D}$ spatial distribution represent the normalized far field differential scattering cross section as a function of the scattering angle.

where $g=\Re\left\{a_{l} b_{l}^{*}\right\} /\left(\left|a_{l}\right|^{2}+\left|b_{l}\right|^{2}\right)$ is the asymmetry parameter in the dipolar regime [37].

Notice that at the first Kerker condition, when the $g$ parameter is maximized in the dipolar regime $g=0.5$, the EM helicity is preserved [see Eq. (10) for $l=1$ ]. In this scenario, it can be inferred from Eq. (12) that there is no radiation in the backscattering direction $(\theta=\pi)$ [6-8]. However, previously deduced in the presence of losses or optical gain, the zero optical backscattering condition cannot emerge as a result of the breaking of the first Kerker condition that imposes both $|\Lambda|<1$ and $g<0.5$. The latter outcome is analyzed in the Supplemental Material [53]. As an illustrative example, we show in Fig. 2 the gradual loss of the zero optical backscattering condition [see Fig. 2(a)], as the absorption rate is increased for a Ge sphere [see Figs. 2(b) and 2(c)]. As can be deduced, it is easy to see how the zero optical backscattering condition is lost in the case of lossy spheres.

Finally, let us briefly analyze the second Kerker condition, given by $a_{1}=-b_{1}$. Let us recall that according to Eq. (10) in the dipolar limit, the EM helicity flips its value from $\langle\Lambda\rangle=+\sigma$ to $\langle\Lambda\rangle=-\sigma$, and the $g$ parameter is minimized, $g=-0.5$, leading to zero optical light scattering in the forward direction [see Eq. (12)]. According to Eq. (1), the second Kerker condition implies both

$$
\sin 2 \alpha_{1}=-\sin 2 \beta_{1} \quad \text { and } \quad \sin ^{2} \alpha_{1}=-\sin ^{2} \beta_{1} .
$$

It is straightforward to notice that lossless spheres, where $\Im\{m\}=0$, cannot satisfy the second Kerker condition since in that scenario $\alpha_{l}, \beta_{l} \in \mathbb{R}$ and, then, the right-hand side of Eq. (13) is unreachable. Interestingly, the second Kerker condition leads to the antiduality condition for dipolar particles, a phenomenon that cannot be achieved for nonactive media $[54,55]$, as we have demonstrated.

Figure 3 illustrates the $g$ parameter for a Ge-like sphere $(m=4)$ versus the size parameter $x=k R$ and the imaginary part of the refractive index contrast $\mathfrak{s}\{m\}$ under plane wave illumination with well-defined helicity $(\sigma=+1)$

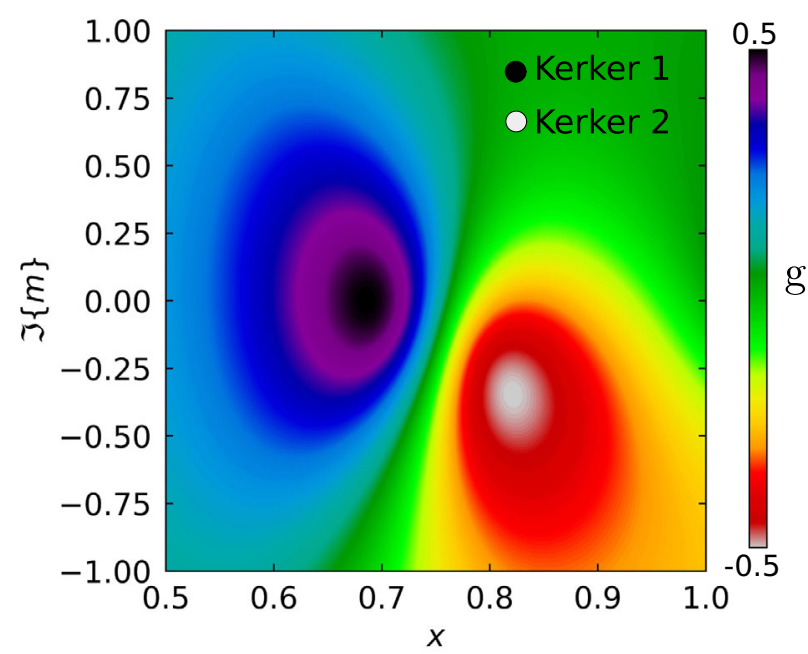

FIG. 3. $g$ parameter vs the imaginary part of the refractive index contrast $\Im\{m\}$ and the $x=k a$ size parameter under well-defined EM helicity $(\sigma=+1)$ plane wave illumination. In this range, the optical response is purely of a dipolar nature. The first and second Kerker conditions are depicted by black and white circles, respectively.

(see Supplemental Material [53]). In this regime, the optical response is almost entirely dipolar and, as a result, the asymmetry parameter is in essence the same magnitude as the expected value of the EM helicity (and $\Lambda_{\pi / 2}$ ), $\langle\Lambda\rangle=2 g$ [33]. As previously mentioned, the first Kerker condition $\left(a_{1}=b_{1}\right)$ arises in a lossless regime $(\Im\{m\}=0)$ at $x \sim 0.675$. This specific size parameter corresponds to the first Kerker condition appearing in Fig. 2(a). As expected, the first Kerker condition does not emerge for $\Im\{m\} \neq 0$. On the other hand, the second Kerker condition does not appear for $\Im\{m\}=0$, in agreement with Eq. (13). In fact, it arises if and only if optical gain is being pumped onto the system. In the particular case of the Ge-like sphere, it emerges for $\Im\{m\} \sim-0.3$ and $x \sim 0.825$, as can be reckoned from Fig. 3.

In conclusion, we have rigorously demonstrated that either losses or optical gain inhibit the appearance of the first Kerker condition for dielectric and plasmonic particles, such as metal spheres. Therefore, in lossy systems, the phenomena associated with the first Kerker condition are greatly modified, and one should carefully analyze the situations where the magnetic and electric extinctions match. As a direct consequence of our analysis, we have shown that the EM duality restoration, identified through the conservation of the EM helicity and, hence, a null optical backscattering condition, cannot be achieved in the presence of losses. In a spin-orbit framework, this phenomenon precludes the full angular momentum exchange from spin to orbit after scattering, inhibiting the emergence of an optical vortex in the backscattering direction. Moreover, recently, massive efforts have been dedicated to maximizing the sensitivity of circular dichroism (CD) 
spectroscopy of chiral molecules [19-25]. Some of these theoretical developments have relied on meeting Kerker's first condition in high index nanoparticles to preserve the helicity of the scattered electromagnetic fields. Our results highlight that special care must be taken into account when considering lossy systems as the first Kerker condition cannot be strictly satisfied.

Furthermore, we have studied the gradual loss of the zero optical backscattering condition for a Ge sphere as the absorption is increased. We have also determined the conditions under which the second Kerker condition emerges and, therefore, the zero forward optical scattering condition is met. The abovementioned statements can be summarized as follows: for the imaginary part of the contrast index $\Im\{m\} \neq 0$, while the second Kerker condition is achievable, the first Kerker condition is inhibited. In contrast, for $\Im\{m\}=0$, the first Kerker condition is obtainable while the second Kerker condition is unreachable. Our analysis unveils an intriguing connection between the Kerker conditions and the energy conservation from fundamental principles, opening new insights into the socalled Mie theory.

This research was supported by the Basque Government (Project No. PI-2016-1-0041 and Fellowship No. PRE2018- 2-0252) and by the Spanish MINECO and MICINN and European Regional Development Fund (ERDF) Projects No. FIS2017-91413-EXP, FIS2017-82804-P, FIS2017-87363-P, PGC2018-095777-B-C21 and Fellowship No. FPU15/ 03566. We acknowledge the PID2019-109905GA-C22 project of the Spanish Ministerio de Ciencia, Innovacion y Universidades (MICIU), Gipuzkoako Foru Aldundia OF23/2019 (ES) project, and Eusko Jaurlaritza Grant No. IT1164-19, and KK-2019/00101.

The authors dedicate this work to the memory of their beloved colleague and friend, Prof. Juan José Sáenz, Mole, who passed away on March 22, 2020.

*jolmostrigo@gmail.com

[1] M. Kerker, D.-S. Wang, and C. Giles, J. Opt. Soc. Am. A 73, 765 (1983).

[2] M. Nieto-Vesperinas, R. Gomez-Medina, and J. J. Saenz, J. Opt. Soc. Am. A 28, 54 (2011).

[3] A. García-Etxarri, R. Gómez-Medina, L. S. Froufe-Pérez, C. López, L. Chantada, F. Scheffold, J. Aizpurua, M. Nieto-Vesperinas, and J. J. Sáenz, Opt. Express 19, 4815 (2011).

[4] A. I. Kuznetsov, A. E. Miroshnichenko, M. L. Brongersma, Y. S. Kivshar, and B. Lukyanchuk, Science 354, aag2472 (2016).

[5] H. C. Hulst and H. C. van de Hulst, Light Scattering by Small Particles (Courier Corporation, New York, 1957).

[6] J.-M. Geffrin, B. García-Cámara, R. Gómez-Medina, P. Albella, L. S. Froufe-Pérez, C. Eyraud, A. Litman,
R. Vaillon, F. González, M. Nieto-Vesperinas, J. J. Sáenz, and F. Moreno, Nat. Commun. 3, 1171 (2012).

[7] Y. H. Fu, A. I. Kuznetsov, A. E. Miroshnichenko, Y. F. Yu, and B. Lukyanchuk, Nat. Commun. 4, 1527 (2013).

[8] S. Person, M. Jain, Z. Lapin, J. J. Sáenz, G. Wicks, and L. Novotny, Nano Lett. 13, 1806 (2013).

[9] J. Olmos-Trigo, D. R. Abujetas, C. Sanz-Fernández, N. de Sousa, J. A. Sánchez-Gil, and J. J. Sáenz, arXiv:2003 .12812 .

[10] B. S. Lukyanchuk, N. V. Voshchinnikov, R. PaniaguaDomínguez, and A. I. Kuznetsov, ACS Photonics 2, 993 (2015).

[11] R. Alaee, R. Filter, D. Lehr, F. Lederer, and C. Rockstuhl, Opt. Lett. 40, 2645 (2015).

[12] Q. Yang, W. Chen, Y. Chen, and W. Liu, arXiv:2004.00418.

[13] K. Ullah, M. Habib, L. Huang, and B. Garcia-Camara, Nanomater. Nanotechnol. 9, 536 (2019).

[14] M. Calkin, Am. Phys. 33, 958 (1965).

[15] J. Olmos-Trigo, M. Meléndez, R. Delgado-Buscalioni, and J. J. Sáenz, Opt. Express 27, 16384 (2019).

[16] L. Wei and F. J. Rodríguez-Fortuño, Phys. Rev. Applied 13, 014008 (2020).

[17] X. Zambrana-Puyalto, I. Fernandez-Corbaton, M. Juan, X. Vidal, and G. Molina-Terriza, Opt. Lett. 38, 1857 (2013).

[18] I. Fernandez-Corbaton, X. Zambrana-Puyalto, N. Tischler, X. Vidal, M. L. Juan, and G. Molina-Terriza, Phys. Rev. Lett. 111, 060401 (2013).

[19] A. García-Etxarri and J. A. Dionne, Phys. Rev. B 87, 235409 (2013).

[20] J. S. Eismann, M. Neugebauer, and P. Banzer, Optica 5, 954 (2018)

[21] L. V. Poulikakos, J. A. Dionne, and A. García-Etxarri, Symmetry 11, 1113 (2019).

[22] J. García-Guirado, M. Svedendahl, J. Puigdollers, and R. Quidant, Nano Lett. 18, 6279 (2019).

[23] J. Feis, D. Beutel, J. Köpfler, X. Garcia-Santiago, C. Rockstuhl, M. Wegener, and I. Fernandez-Corbaton, Phys. Rev. Lett. 124, 033201 (2020).

[24] F. Graf, J. Feis, X. Garcia-Santiago, M. Wegener, C. Rockstuhl, and I. Fernandez-Corbaton, ACS Photonics 6, 482 (2019).

[25] J. Lasa-Alonso, D. R. Abujetas, A. Nodar, J. A. Dionne, J. J. Saenz, G. Molina-Terriza, J. Aizpurua, and A. Garcia-Etxarri, arXiv:2003.07653.

[26] C. Schwartz and A. Dogariu, Opt. Express 14, 8425 (2006).

[27] G. Tkachenko and E. Brasselet, Nat. Commun. 5, 1 (2014).

[28] K. Y. Bliokh, F. Rodríguez-Fortuño, F. Nori, and A. V. Zayats, Nat. Photonics 9, 796 (2015).

[29] M. Rafayelyan, G. Tkachenko, and E. Brasselet, Phys. Rev. Lett. 116, 253902 (2016).

[30] F. Alpeggiani, K. Y. Bliokh, F. Nori, and L. Kuipers, Phys. Rev. Lett. 120, 243605 (2018).

[31] S. Nechayev, J. S. Eismann, G. Leuchs, and P. Banzer, Phys. Rev. B 99, 075155 (2019).

[32] D. R. Abujetas and J. A. Sánchez-Gil, ACS Photonics 7, 534 (2020).

[33] J. Olmos-Trigo, C. Sanz-Fernández, F. S. Bergeret, and J. J. Sáenz, Opt. Lett. 44, 1762 (2019).

[34] M. Nieto-Vesperinas, J. Sáenz, R. Gómez-Medina, and L. Chantada, Opt. Express 18, 11428 (2010). 
[35] R. Gómez-Medina, B. García-Cámara, I. Suárez-Lacalle, L. Froufe-Pérez, F. González, F. Moreno, M. Nieto-Vesperinas, and J. Sáenz, Photonics Nanostruct. Fundam. Appl. 10, 345 (2012).

[36] X. Xu, M. Nieto-Vesperinas, C.-W. Qiu, X. Liu, D. Gao, Y. Zhang, and B. Li, Laser Photonics Rev. 14, 1900265 (2020).

[37] R. Gómez-Medina, L. S. Froufe-Pérez, M. Yépez, F. Scheffold, M. Nieto-Vesperinas, and J. J. Sáenz, Phys. Rev. A 85, 035802 (2012).

[38] R. R. Naraghi, S. Sukhov, and A. Dogariu, Opt. Lett. 40, 585 (2015).

[39] P. Varytis and K. Busch, Opt. Express 28, 1714 (2020).

[40] G. Araneda, S. Walser, Y. Colombe, D. Higginbottom, J. Volz, R. Blatt, and A. Rauschenbeutel, Nat. Phys. 15, 17 (2019).

[41] J. Olmos-Trigo, C. Sanz-Fernández, A. García-Etxarri, G. Molina-Terriza, F. S. Bergeret, and J. J. Sáenz, Phys. Rev. A 99, 013852 (2019).

[42] M. Kerker, Appl. Opt. 17, 3337 (1978).

[43] A. Alù and N. Engheta, J. Nanophoton. 4, 041590 (2010).

[44] W. Liu and Y. S. Kivshar, Opt. Express 26, 13085 (2018).

[45] J. Olmos-Trigo, D. R. Abujetas, C. Sanz-Fernández, J. A. Sánchez-Gil, and J. J. Sáenz, Phys. Rev. Research 2, 013225 (2020).
[46] A. Pors, S. K. Andersen, and S. I. Bozhevolnyi, Opt. Express 23, 28808 (2015).

[47] M. Decker and I. Staude, J. Opt. 18, 103001 (2016).

[48] D. Gao, W. Ding, M. Nieto-Vesperinas, X. Ding, M. Rahman, T. Zhang, C. Lim, and C.-W. Qiu, Light Sci. Appl. 6, e17039 (2017).

[49] J. Olmos-Trigo, C. Sanz-Fernández, D. R. Abujetas, A. García-Etxarri, G. Molina-Terriza, J. A. Sánchez-Gil, F. S. Bergeret, and J. J. Sáenz, J. Appl. Phys. 126, 033104 (2019).

[50] Note that the argument $m x=m_{p} k_{0} R$ does not depend on the refractive index of the external medium.

[51] G. N. Watson, A Treatise on the Theory of Bessel Functions (Cambridge University Press, Cambridge, England, 1995).

[52] A. Aiello and M. Berry, J. Opt. 17, 062001 (2015).

[53] See Supplemental Material at http://link.aps.org/ supplemental/10.1103/PhysRevLett.125.073205 for an analytical analysis of the asymmetry parameter behavior in the vicinity of the first Kerker condition when losses or optical gain are considered.

[54] X. Zambrana-Puyalto, X. Vidal, M. L. Juan, and G. Molina-Terriza, Opt. Express 21, 17520 (2013).

[55] P. Gutsche and M. Nieto-Vesperinas, Sci. Rep. 8, 9416 (2018). 